\title{
An Undergraduate Computational Aerodynamics Curriculum
}

\author{
Keith Bergeron, Russell Cummings, Capt Robert \\ Decker, Maj Jacob Freeman, Capt Charlie Hoke, \\ and Jurgen Seidel \\ US Air Force Academy (USAFA), Colorado \\ Springs, $C O$ \\ $\{$ keith.bergeron, russell.cummings, robert.decker, \\ jacob.freeman, charlie.hoke, \\ jurgen.seidel\}@usafa.edu
}

\begin{abstract}
Modeling and Simulation (M\&S) as part of the Aeronautical Engineering major at the United States Air Force Academy (USAFA) has grown from a one course introduction to an integrated and essential component for developing future aerospace leaders. This paper documents the progress the USAFA Department of Aeronautics (DFAN) has made since 2003 to teach cadets, through a 2-course sequence, how to gain an understanding of aerodynamic phenomena using computational methods made possible with Department of Defense (DoD) High Performance Computing Modernization Program (HPCMP) resources. The first course in of the sequence builds upon demonstrations, made in early core coursework, to relatively simple applications and reinforcement of introductory fluid aerodynamics. The second course further develops the "intelligent users" of computational fluid dynamics (CFD) by working as teams on current USAF research projects. Cadet projects have included participation on the C2D Challenge Project, study of plasma actuators, comparison of wake characteristics for NASA's Crew Exploration Vehicle (CEV), and drag validation simulations for the C-130P. These projects made extensive use of high performance computing (HPC) resources at the Alaska Regional Supercomputing Center (ARSC) as well as Maui High Performance Computing Center (MHPCC). Besides the external validation of project sponsors, the curriculum has received very high student satisfaction on End-of-Course evaluations comparing well with the highest rated courses at USAFA.
\end{abstract}

\author{
Scott A. Morton and David M. McDaniel \\ US Air Force SEEK EAGLE Office (46 SK/SKI), \\ Eglin $A F B, F L$ \\ \{scott.morton, david.mcdaniel.ctr\}@eglin.af.mil
}

\section{Introduction}

United States Air Force (USAF) aeronautical engineers are strongly involved in the national commitment of maintaining global air superiority through the development of state-of-the-art aircraft for the USAF. The Aeronautics department at USAFA contributes actively to this commitment by preparing cadets for service to the Air Force as skilled entry-level aeronautical engineers with competencies in six disciplines: aerodynamics, aircraft and aircraft engine design, aerospace materials and structures, propulsion, aircraft flight mechanics, and experimental and computational investigation. Specifically, the purpose of the aerodynamics discipline is to teach cadets how and why airplanes fly. With the foundations of flight and aerodynamics initially studied in the engineering courses, Fundamentals of Aeronautics and Aero-Thermodynamics, cadets acquire knowledge on the principles of aerodynamics, fluid mechanics, and gas dynamics with regard to flow physics of solid objects in flight. Until 2003, these two courses were followed by a standard 3course sequence in Aeronautical Fluid Dynamics, Aerodynamics, and Advanced Aerodynamics for all majors. Finally, electives within the aerodynamics curriculum included Introduction to Hypersonics and Advanced Applied Aeronautics. However, the turn of the century heralded a new paradigm in aerodynamics education at USAFA to match the revolutions well underway in aerodynamics research - computational fluid dynamics (CFD) and high performance computing (HPC). 
While USAFA researchers had been active in the High Performance Computing Modernization Program (HPCMP) since the mid 1990s, the opportunity to move this innovative way of solving science and engineering problems into the Academy's undergraduate curriculum received several kick starts between 2000 and 2001. ${ }^{[1]}$ During their visits, Dr Roche, Secretary of the Air Force, and Dr Schwartz, Director of the Air Force Office of Scientific Research (AFOSR), noted a lack of modeling and simulation (M\&S) emphasis within the USAFA curriculum. To address this need, AFOSR signed a Memorandum of Agreement with the Academy leading to the creation of the Modeling and Simulation Research Center (M\&SRC). The M\&SRC's initial goals included building on the USAFA Department of Aeronautics (DFAN) CFD strengths and increasing the basic and engineering research in $M \& S$. The center is formally manned by a director and systems administrator, and currently has the following computing resources:

- Dual-use Research and Academic remotelyaccessible cluster composed of

- 164 CPU Xeon nodes

- $56 \mathrm{CPU}$ dual-core Opteron nodes

- Common 10Tb RAID storage

- 4GB RAM/node

- Post-processing (visualization) workstations

- Two 4 CPU nodes with 16 GB RAM

- SGI Global Shared Memory Altix B4700

- 64 Itanium CPUs; 512GB RAM

- 70Tb RAID common storage

- Direct DREN link to OC-12

The M\&SRC also provides support for floating researchers who are assigned to USAFA departments through a competitive selection process. Two current researchers reside in DFAN and the Department of Physics. Through this arrangement departments may leverage their M\&S and HPC research programs and increase cadet educational opportunities.

Based on these events and the desire to continue its long history of developing innovative educational concepts and programs to better prepare aeronautical engineers, DFAN began the development of a computational aerodynamics curriculum. To accomplish this effort, several changes were required. Approximately, half of the Aerodynamics classes were deleted from the course and shuffled among AeroThermodynamics, Aeronautical Fluid Dynamics, and Advanced Aerodynamics. The empty lessons in Aerodynamics were then filled with computational fluid dynamics as outlined in Section 3. Ultimately, the end goal is to help cadets gain a better understanding of $M \& S$ and HPC, such that after graduation they can make more informed decisions and contributions for their USAF and the Department of Defense (DoD) units of assignment.

\section{Objective}

A critical piece to making this vision a reality was the conscious and deliberate decision to focus the education on developing intelligent users of CFD. Though there is an ongoing discussion to determine if the education that aerospace engineering students are receiving in terms of structured programming and computation tools matches the needs of employers ${ }^{[2]}$, the Academy has unique conditions placed on the cadets' educational experience which make the goal of developing CFD programmers less practical than in non-military undergraduate institutions. First and foremost, is the limited amount of time cadets has to accomplish a demanding programeach cadet must complete their degree program in four years. Additionally, the USAFA education includes a strong core curriculum component designed to educate the "whole-person". ${ }^{[3]}$ The core courses represent 102 semester hrs of the total $147 \mathrm{hrs}$ to graduate with an ABET accredited Bachelor of Science in Aeronautical Engineering. As a result, there are very limited opportunities for formal programming courses unless cadets have validation or transfer credits.

Fortuitously, DFAN has been able to capitalize on a couple of opportunities in order to address these constraints. In particular, the Academy aeronautics program incorporates a strong MATLAB foundation across many courses. Care is taken to focus on the programming constructs, problem solving strategies, algorithms, and data structures available in MATLAB to reinforce programming fundamentals over an extended period of time. Also, the strong experimental and flight test relationship DFAN enjoys with the Air Force Research Laboratories, USAF commands, and several DoD organizations encourages a student-centered learning focus where cadets learn the value of properly using existing tools and methods to provide answers to complex problems in a limited amount of time. Cadets learn not only the importance of scoping their work but also the importance, via "hands-on" experiences, of how a detailed study will contribute to the larger effort. Therefore, the objective of the DFAN computational aerodynamics curriculum is to have cadets demonstrate an understanding of numerical methods as used for CFD calculations such that they may plan, collect and analyze data, and draw appropriate conclusions from computational investigations.

\section{Methodology}

Figure $1^{[9]}$ illustrates the overall "integration" concept used by DFAN to incorporate computational aerodynamics into the DFAN curriculum. 
In their early coursework, cadets see CFD from a demonstration perspective where they learn basic aerodynamic concepts and have a chance to "play" with these concepts. While AE 341, Aeronautical Fluid Dynamics, covers the traditional topics including use of the integral momentum equation to experimentally determine the drag acting on a cylinder in a low-speed stream, a key element of the course is the use of MATLAB to investigate unsteady Poiseuille flow and to compute a steady, laminar boundary layer. While using MATLAB has not traditionally been considered within the paradigm of CFD, this experience provides essential preparation for $\mathrm{AE} 342$ and represents a valuable tool which can be used to develop introductory programming skills.

AE 342, Computational Aerodynamics ${ }^{[9]}$, serves as a transitional course, and it, like AE 341, is taught to all junior-level Aeronautics majors. The course is the first formal introduction to the basics of computational aerodynamics, including numerical methods, grid generation, post-processing, and best practices in simulating aerodynamics with CFD. The course follows a four-step CFD process as illustrated in Figure 2.

Software used for these steps include Gridgen by POINTWISE ${ }^{\circledR}$, Cobalt, Tecplot, and Intelligent Light's Fieldview. Emphasis is place on the flow solution and post processing components of the process not on developing/optimizing code. In fact, the Cooperative Research and Development Agreement (CRADA) between the Academy and Cobalt, LLC, provide a convenient and flexible avenue to pursue such code development. For example, cadets have played a significant role in the development of a body forcing capability within Cobalt (Ref. Section 4). AE 342 course outcomes include: understanding aerodynamic concepts such as lift, drag, vortical flows; viscous effects, and shock waves, generating structured two-dimensional (2D) and unstructured (2D, three-dimensional [3D]) grids around moderately complex shapes; performing a CFD calculation, analyzing the results from a CFD calculation using graphical and non-graphical techniques; and producing accurate written, oral, and graphical communication. Pre- and post- analyses of results are emphasized throughout the course and in each of the 8 assigned projects. The first four course projects continue the use of MATLAB as in AE 341, but subsequent projects require cadets to develop such specific skills as determining appropriate grid spacing, performing grid refinement and time-resolution studies, determining level of CFD tools required, and identifying relevant flow features. It is absolutely critical in this paradigm of educating future aeronautical engineers that cadets develop expertise in their solutions and appreciation for the limitations of those solutions such that they are confident they are not just making colorful graphics. The course projects have also been used and adapted for visiting researchers with great success. All computations and analysis are completed on local resources as listed in Section 1. One of the major products of AE 342 is to provide cadets with the skills to transition to the use of the Major Shared Resource Centers and the Allocated Distributed Centers resources. These skills are best developed in a more benign, user friendly environment than on massively parallel machines.

As their computational proficiency increases, cadets are led to applications and more structured exploration in their senior-level coursework. Cadets are able to take their CFD knowledge and apply it in aircraft design (AE 481/482) and elective courses such as independent research projects or the newly created AE 447, Advanced Computational Aerodynamics. AE 447 was first offered in Fall 2007 with an enrollment of nine cadets. The course sought to build on the success of AE 342 and parallel the aeronautical major's experimental methods course, AE 471, Aeronautics Laboratory. DFAN recognized early the pivotal role computational investigations are playing in contemporary research, and actively looked for a mechanism to strengthen the experimental-computational synergy. Therefore, AE 447 is designed as a team-oriented course in which two cadets are grouped with a computational research mentor. Projects are picked to include in-depth investigation of unsteady flows, boundary layers, turbulence models, shocks, and multi-physics simulations and where possible correspond to an ongoing DFAN experimental or flight test effort. For the Fall 2007 offering, there were five specific projects picked from within these major categories: F-16 Stability and Control Modeling, C-130P Drag Reduction Analysis, Aero-Servo-Optics, Free Shear Layer Simulation, and a Flow Solver Comparison Study. These projects followed a well-defined test plan process and require periodic formal briefings to an instructor team which included a senior leader/project manager/sponsor. The intent was to reinforce project organizational and communication skills also addressed in AE 471. Most cadets take AE 447 after completing $\mathrm{AE} \mathrm{471,} \mathrm{but} \mathrm{there}$ has been one case in which a cadet was dual enrolled in $\mathrm{AE} 447$ and $\mathrm{AE} 471$ working on the same problem for each course but with different methods of investigations. Each team briefed their final report and prepared a manuscript for possible conference presentation.

Specific course objectives build on the foundations

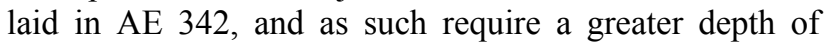
knowledge and analysis. Grid generation, boundary conditions, and turbulence modeling are covered in more detail, and several in-class laboratory sessions are dedicated to each topic. Again, the increased complexity of the course is reflected in the computational resources required. All projects are associated with active HPC projects such that cadets must use machines located at one 
of the MSRCs or ADCs. The success of the course is due to the support of these centers and the high quality of mentors for each project.

\section{Results}

The computational aerodynamics curriculum has not developed without some growing pains, but by several measures the AE 342 and AE 447 courses are among the highest rated not only in the Aeronautics Department but across all courses taught at USAFA. Additionally, the praise of our research sponsors and our success at placing both a cadet and a doctoral student into HPC focused graduate programs attests to the value of the USAFA program.

\subsection{Intermediate CFD Skill Development}

The initial offering of AE 342 in 2003 proved to be slightly ambitious. The number and scope of topics was greater than the time most cadets devoted to the class. Not only did this reduce the amount of material covered, but it also led to a relatively low satisfaction rating on the cadet end-of-course (EOC) evaluations. However, a major contribution to this problem was the unexpected lack of cadet experience with command line and UNIX environments. Therefore, in addition to better scoping of assignments, an extensive UNIX tutorial was created for the Fall 2004 offering, and these changes led to a significant improvement in the efficiency and completion of assignments. The following year a dedicated "laboratory" time of one hour after each class was added to allow cadets the opportunity to work on projects with their instructor immediately following the day's presentation. Again, a considerable improvement in the quality and timeliness of completed assignments was realized. During 2006 and 2007, the course appears to have reached a steady state and measures of cadet performance continue to rise as do EOC feedback results.

\subsection{Advanced CFD with HPC}

The first offering of AE 447 had many factors working in its favor. First, the experiences learned from AE 342 emphasized the need to properly scope the work. Second, using AE 471 as a template for course tempo and structure, in addition to cadet familiarity with the course structure, allowed potential problems to be identified early. Third, the responsiveness and professionalism of the ARSC staff kept the cadets and their projects on track in a very dynamic academic/research environment. Fourth, the dedicated mentors teamed with all of the cadets provided practically $24 / 7$ help for the cadet teams. Each research mentor has had years of experience in not only CFD but also as mentors for AE 471 projects. Fifth, and maybe most importantly, the cadets chose AE 447 as elective - the cadets were highly motivated!

Three of the $5 \mathrm{AE} 447$ projects were presented at the American Institute for Aeronautics and Astronautics (AIAA) Student Regional Conference in St Louis, April 2008:

- "Maneuver Parameter Study for the Computational Modeling of a Fighter Aircraft" $(\mathrm{SMHM})^{[4]}$

- "Computational Study of the Effect of Plasma Actuators on Circular Cylinder Wakes" (RJS) ${ }^{[5]}$

- "C-130P „Combat Shadow" Drag Validation Using CFD" (DSH) ${ }^{[6]}$

In addition, a combined CFD and experiment paper was presented which was the result of a Cadet Summer Research Project at Johnson Space Center (JSC), "Aerodynamic Comparison of Crew Exploration Vehicle and Parachute Test Vehicle Wake Characteristics" (MY) ${ }^{[7]}$ While working at JSC, the research sponsors learned that the cadet had an introductory CFD background based on his enrollment the previous Spring in $\mathrm{AE} \mathrm{342.} \mathrm{The} \mathrm{sponsors} \mathrm{asked} \mathrm{the} \mathrm{cadet,} \mathrm{if} \mathrm{he} \mathrm{felt}$ comfortable using his CFD background on the CEV project. The cadet responded in the affirmative, and the resulting CFD analysis using HPC resources proved to be an important component of the final results. A short synopsis and example result from each project follows. In each case cadets made significant contributions, which might not have been otherwise possible, by using and integrating mature HPC CFD tools into their research.

\section{3. "Maneuver Parameter Study for the Computational Modeling of a Fighter Aircraft" ${ }^{[4]}$}

Cadets in this project worked directly with researchers on the C2D Challenge Project. Their work documents the production of a simulated atypical aircraft maneuver-or training maneuver (ref Figure 3) - used to generate a nonlinear model that can predict the aerodynamic forces acting on the aircraft during realistic maneuvers at the same flight condition. Only rotational motion in the longitudinal axis (pitch) was considered. Three parameter studies were conducted concerning the training maneuver-variations with respect to time, frequency, and angle of attack growth rate. When compared with results from a simulation where the aircraft motion was prescribed based on an actual pitch doublet test maneuver, the lift coefficient prediction models were more than $99 \%$ accurate. The drag coefficient prediction models were approximately $94 \%$ accurate. The parameter study revealed that while increasing the input signal time and frequency increases the resulting model's accuracy, increasing the growth rate 
decreases the model's ability to accurately predict aerodynamic forces during maneuvers similar to a pitch doublet. Overall, the cadet's method showed promise for producing low-order nonlinear aerodynamic models of actual flight maneuvers, and could potentially save a significant amount of time and money by reducing the dependency on current flight test methods.

\section{4. "Computational Study of the Effect of Plasma Actuators on Circular Cylinder Wakes"[5]}

For this project, cadets conducted volume forcing simulations to model plasma actuation on the flow around a circular cylinder (ref Figure 4) in cross flow at a Reynolds number of 6,500 were performed. Since no practical computational simulation of plasma actuators exists, the actuators were replaced with a volume force to eliminate the difficulties associated with simulating plasma physics. Therefore, the study investigated the magnitude of the volume force coefficient associated with experimental investigations. Unforced simulations were performed to determine the baseline flow field, and the results matched previous computational and experimental studies. Simulations with a constant volume force acting normal and tangential to the cylinder surface with a force coefficient of $\mathrm{f}_{\mathrm{B}}=1.23 * 10^{5} \mathrm{Nm}^{-3}$ were also performed, and the volume forcing was effective in simulating plasma actuation. Tangential forcing resulted in a 30 percent drag reduction, while normal forcing caused a 5 percent drag increase. Key to this work was the integration of computational and experimental results.

\section{5. "C-130P 'Combat Shadow' Drag Validation Using CFD"[6]}

Cadets involved with this project had the unique opportunity to work with an operational unit, the Air Force Special Operations Command (AFSOC). AFSOC asked USAFA to validate drag increments incurred on the $\mathrm{C}-130 \mathrm{P}$ and to develop a reliable and expeditious method to analyze future modifications. The $\mathrm{C}-130 \mathrm{P}$ experiences increased excrescent drag due to wing tanks and refueling pods (similar to the $\mathrm{C}-130 \mathrm{H}$ model), and a suite of sensors and antennae clustered around the nose. Figure 5 illustrates these geometry affects. While wind tunnel experimentation and flight test have already been accomplished for the C-130P, AFSOC is interested in the comparison of computational accuracy to experimental and operational data points. A validated viscous grid, high-fidelity Navier-Stokes simulation was completed using a DES-SARC turbulence modeling to replicate fullscale flight conditions at angles of attack from $2^{\circ}$ to $12^{\circ}$ with a Reynolds number of $2.9 \times 10^{7}$ and a Mach number of 0.4. At low angles of attack (cruise conditions) the C-
$130 \mathrm{P}$ antennae and sensors create a $2.1 \%$ drag increase, with data points differing from flight test results by less than $6 \%$. Scaled cases $\left(1 / 48^{\text {th }}\right)$ simulations made in conjunction with wind tunnel tests showed a similar drag increase at a Reynolds number of $6.4 \times 10^{5}$. The computational methods only varied from the experimental methods by $1.5 \%$. This small error, coupled with quick turn-around times at low cost, establishes CFD at USAFA as viable option to analyze future $\mathrm{C}-130$ variants.

\section{6. 'C-130P 'Combat Shadow' Drag Validation Using CFD", ${ }^{[7]}$}

NASA's Crew Exploration Vehicle (CEV) will return to earth under parachutes similar to the Apollo spacecraft. In order to test the parachute system, a series of drop tests must be completed. Since the full sized capsule is too large to fit in the cargo aircraft used for drop tests, a version of the capsule with an abbreviated after-body known as the Parachute Test Vehicle (PTV) was designed. This effort analyzed the differences in the wake of the CEV and PTV at speeds and orientations that correspond to various parachute deployment phases. As in sections 4.4 and 4.5 , both experimental and computational fluid dynamics comparisons were made as seen in Figure 6. In particular, a turbulence model study was conducted at wind tunnel Reynolds numbers, and a comparison to experimental data determined that the Spalart Allmaras Rotation Correction Detached Eddy Simulation (SARC-DES) most accurately modeled the wake characteristics. The direct comparison of PTV and CEV experimental data found a maximum average difference in the wake was $4.4 \%$. The wake of the PTV tended to be about $8.5 \%$ larger than the CEV wake at the distance of 5.5 diameters aft of the vehicle and was essentially the same at a distance of 6.5 diameters.

The cadet presentations at the AIAA Regional Conference went very well - the Maneuver Study in section 4.3 placed 2nd and the CEV work of section 4.6 placed 3rd. The top 3 places are the only rankings, but the feedback from the evaluators for the other cadet presentations was also very positive.

\subsection{Cadet End of Course (EOC) Feedback}

At the end of every semester cadets complete a 23 question course report. The report is partitioned into 3 sections: Diagnostic Feedback for the Instructor (questions 1-12), Information about the Course (13-19), and General Evaluation (20-23). Figure 7 shows the EOC report for AE 447, Fall 2007. 


\section{Significance to DoD}

The EOC results, AIAA presentation success, and the feedback from the research sponsors for each of the subprojects indicate that USAFA has the basis of a very successful HPC and M\&S program. In addition to making significant DoD research contributions through the results gained from the cadet projects, the program is also providing officers, both cadets and instructors, who have substantial skills in M\&S and HPC and who will thus make better leaders.

\section{Systems Used}

ARSC: Midnight, 2,312 compute processors Opteron Sun cluster and Iceberg, 5 teraflop Power4 IBM. MHPCC: Jaws, 5,120 (1,280 nodes) processor Dell PowerEdge 1955 blade server cluster.

\section{Computational Technology Area}

Computational Fluid Dynamics (CFD)

\section{Acknowledgments}

Funding for this work was provided by the Air Force Seek Eagle Office, AFOSR, and NASA. Many of the calculations were performed using computer resources granted by the DoD HPCMP C2D Challenge Project.

\section{References}

1. Cummings, R.M., S.A. Morton, and K. Bergeron, "Modeling and Simulation Research and Instruction at the U.S. Air Force Academy." AIAA Paper 2004-4935, 2004.

2. Arlington, A., Survey for CFD managers, AIAA New Initiatives Subcommittee of the Technical Activities Committee, 2008.

3. Taylor, H., 2007 USAFA Curriculum Handbook, HQ USAFA/DFRC, USAF Academy, CO, 2007.

4. Schwartz, M.S., L.L. Matthews, C.M. Hoke, and D.R. McDaniel, "Maneuver Parameter Study for the Computational Modeling of a Fighter Aircraft." 2008 AIAA Student Regional Conference, St. Louis, 2008.

5. Russo, P., S. Javaid, and J. Seidel, "Computational Study of the Effect of Plasma Actuators on Circular Cylinder Wakes." 2008 AIAA Student Regional Conference, St Louis, 2008.

6. DeNeve, A.J., A.J. Seifert, and C.M. Hoke, "C-130P 'Combat Shadow' Drag Validation Using CFD.” 2008 AIAA Student Regional Conference, St. Louis, 2008.

7. Moorman, C. and T. Yechout, "C-130P 'Combat Shadow' Drag Validation Using CFD.” 2008 AIAA Student Regional Conference, St. Louis, 2008.
8. "Course Report, AE447", HQ USAFA/DFR, USAF Academy, CO, 2007.

9. Cummings, R. and Morton, S., "Computational Aerodynamics Goes to School: A Course in CFD for Undergraduate Students", AIAA Paper 2005-1072, 2005.

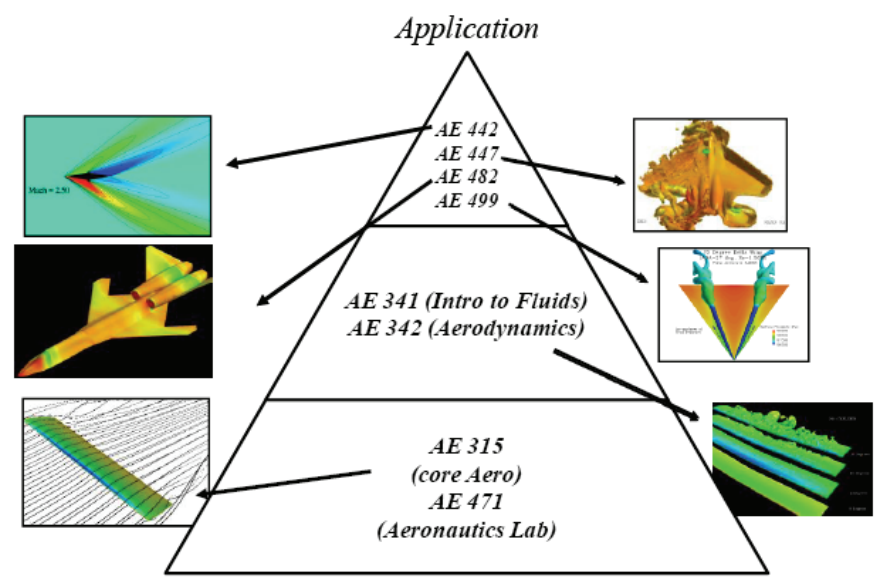

Demonstration

Figure 1. Computational aerodynamics integration into department courses. Note: AE 442=Advanced Aerodynamics, AE 447=Advanced Computational Aerodynamics (elective), AE 482=Aircraft Design, AE 499=Independent Study (elective).

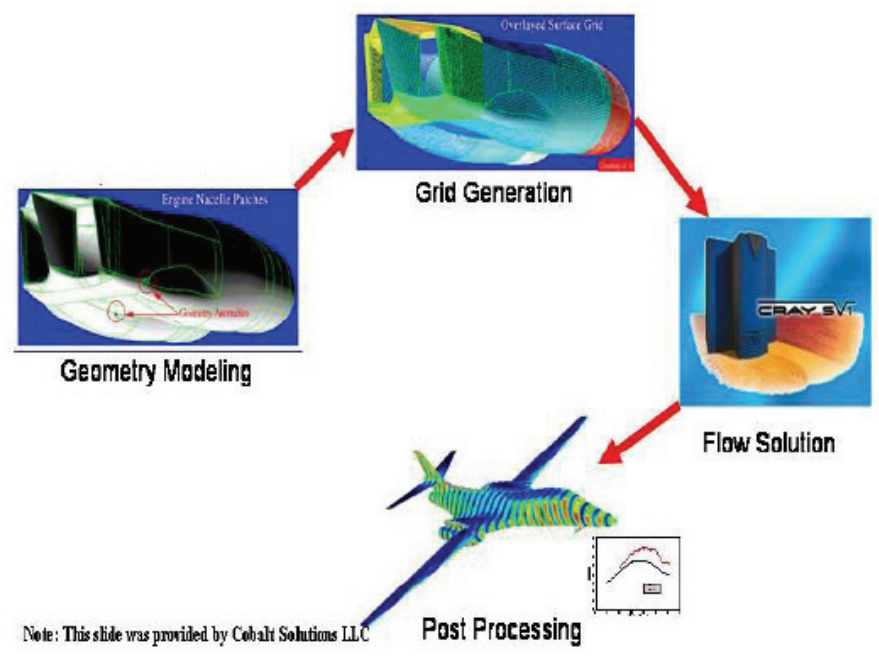

Figure 2. CFD four-step process taught in AE342 ${ }^{[9]}$ 


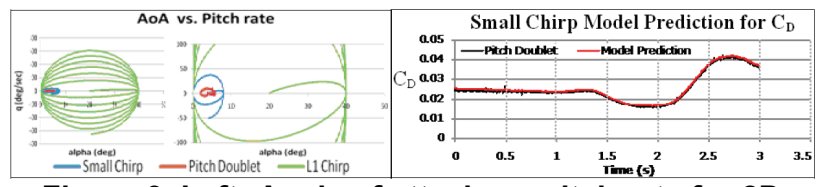

Figure 3. Left: Angle of attack vs. pitch rate for 2D chirp, small chirp, and pitch doublet. Right: Results of small chirp model prediction for pitch doublet. ${ }^{[4]}$

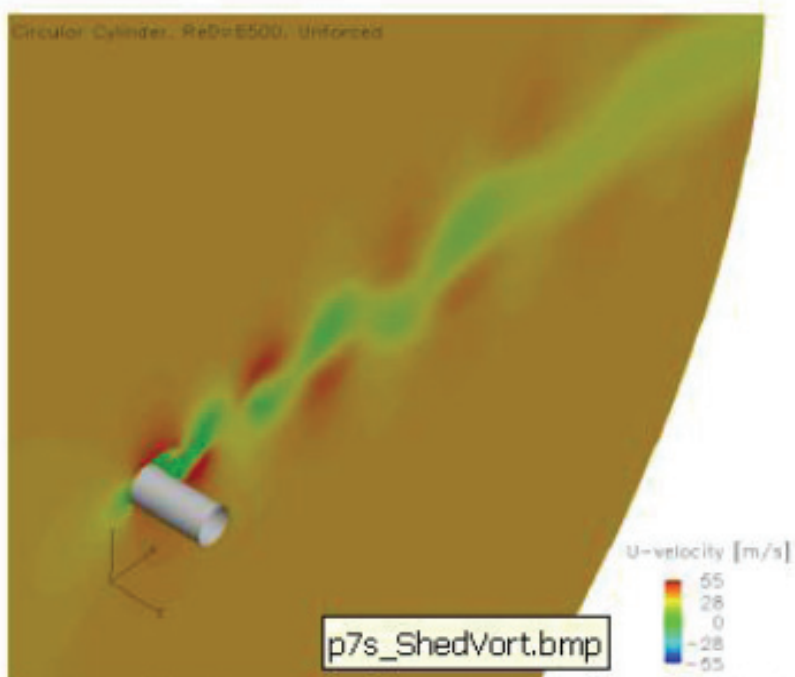

Figure 4. Circular cylinder unforced wake, U-velocity, iteration $=11,420^{[5]}$

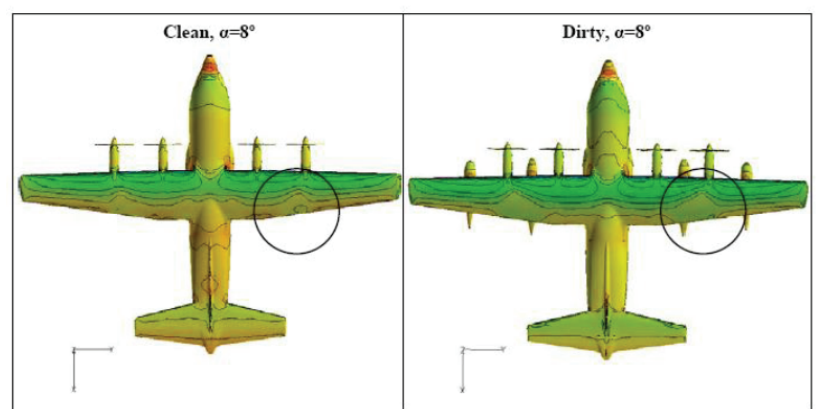

Figure 5. Pressure contours on the full-scale clean (left) and dirty (right) $\mathrm{C}-130$. The circled regions show the area of interest with an increased area of separated flow on dirty model (right) ${ }^{[6]}$
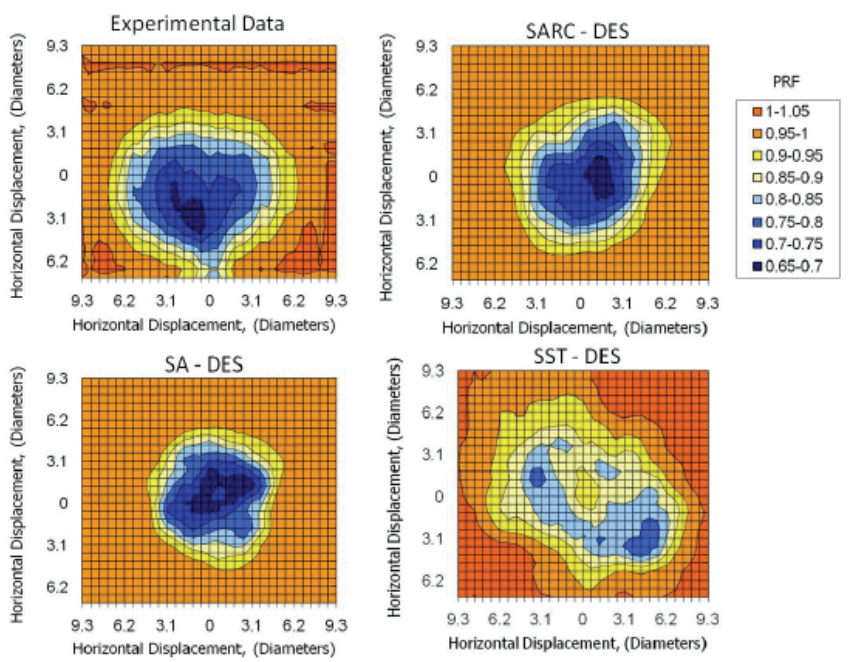

Figure 6. Results of small chirp model prediction for pitch doublet $^{[7]}$
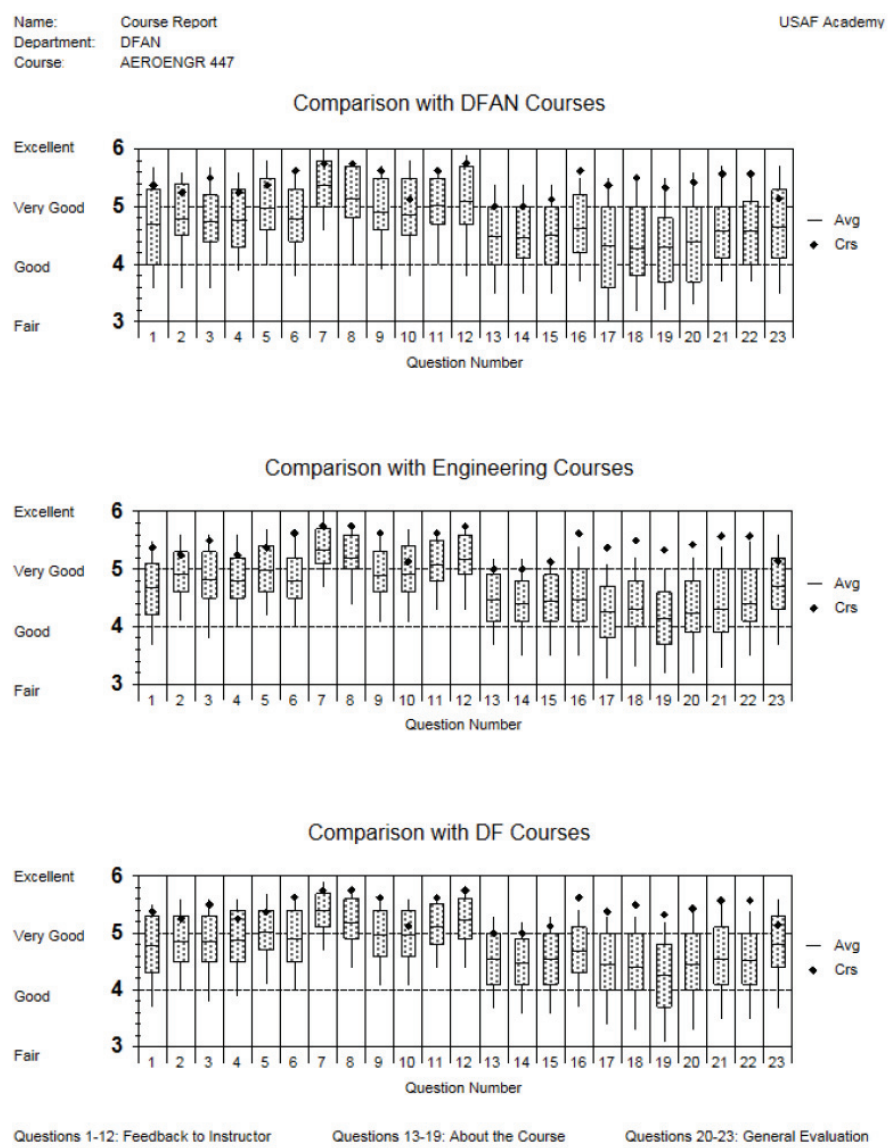

Figure 7. Sample question of the Cadet EOC feedback form $^{[7]}$ 